\title{
Sinusitis Maxilar por Osteonecrosis Asociada a Bisfosfonatos en Pacientes con Cáncer de Mama: serie de Casos
}

\author{
Maxillary Sinusitis Associated to Bisphosphonate-Related \\ Osteonecrosis in Patients with Breast Cancer: Case Series
}

Figueroa, L.;; Sáez, F."; Alvarado, B."; Gallardo, P.* \& Cifuentes, J.**

FIGUEROA, L.; SÁEZ, F.; ALVARADO, B.; GALLARDO, P. \& CIFUENTES, J. Sinusitis maxilar por osteonecrosis asociada a bisfosfonatos en pacientes con cáncer de mama: Serie de casos. Int. J. Odontostomat., 9(3):449-455, 2015.

RESUMEN: Los bisfosfonatos (BF) son fármacos ampliamente utilizados como estabilizadores óseos en el tratamiento de metástasis óseas, osteoporosis, enfermedad de Paget, entre otras patologías, debido a sus efectos anti-tumorales y a la característica de inhibir la actividad osteoclástica. La osteonecrosis maxilar asociada a BF, hoy en día osteonecrosis maxilar asociada a fármacos (ONMF) es definida como la presencia de hueso expuesto, no-vascularizado y necrótico en la cavidad oral por un periodo mayor a ocho semanas, con una historia positiva de tratamiento con fármacos anti-reabsorción ósea (BP, inhibidores del ligando RANKL) y/o anti-angiogénicos y sin antecedentes de tratamiento con radiación o metástasis obvia en los maxilares. La frecuencia de ONMF es incierta. La mandíbula es más frecuentemente afectada por ONMF que el maxilar. Pocos casos de ONMF en el maxilar han sido descritos con un diagnostico de sinusitis maxilar simultáneo. Tres casos con sinusitis maxilar asociada a ONMF son presentados en este trabajo. Todos los pacientes fueron mujeres con una historia positiva de cáncer de mama y tratamiento con bisfosfonatos. Los primeros dos casos, desarrollaron ONMF después de una extracción del tercer molar maxilar. El tercer caso con ONMF en el maxilar, sólo tenía antecedentes de curetaje periodontal. Una tomografía computada fue realizada y mostró compromiso del seno maxilar en todos los pacientes. Modalidades diagnósticas para evaluar la extensión de la necrosis y el compromiso del seno, como también alternativas de tratamiento son descritas en este estudio. Finalmente, una revisión actualizada de la literatura es presentada.

PALABRAS CLAVE: bisfosfonatos, osteonecrosis maxilar asociada a fármacos, sinusitis.

\section{INTRODUCCIÓN}

Los Bisfosfonatos (BF) son fármacos ampliamente utilizados como estabilizadores óseos en el tratamiento de metástasis óseas, osteoporosis, enfermedad de Paget, entre otras patologías, debido a sus efectos anti-tumorales y a la característica de inhibir la actividad osteoclástica (Abu-ld et al., 2008). Esta característica está dada por un lado por la disminución del reclutamiento e inhibición de la función del osteoclasto. Por otro lado, inducen su apoptosis, disminuyendo finalmente el recambio óseo (Reszka \& Rodan, 2007). Algunos de los efectos anti-tumorales descritos para los BF son: inhibir la invasión y la adhesión a la matriz extracelular de células tumorales, inhibir las metaloproteinasas y la angiogénisis (Gutta \& Louis, 2007).
El cambio de término de osteonecrosis asociada a BF por osteonecrosis maxilar asociada a fármacos (ONMF), se ha propuesto para justificar el número creciente de casos con osteonecrosis donde los maxilares han sido involucrados asociados a otro tipo de fármacos: antireabsorción ósea (como bisfosfonatos, inhibidores del ligando RANKL) y fármacos antiangiogénicos (Ruggiero et al., 2014). La ONMF, es definida como la presencia de hueso expuesto, novascularizado y necrótico en la cavidad oral por un periodo mayor a ocho semanas, con una historia positiva de tratamiento con fármacos anti-reabsorción ósea y/o anti-angiogénicos y sin antecedentes de radioterapia en la región de cabeza y cuello (Ruggiero et al., 2014, 2009, 2004).

* Staff. Servicio de Cirugía y Traumatología Oral y Maxilofacial, Clínica Alemana, Santiago, Chile.

* Residente. Servicio de Cirugía y Traumatología Oral y Maxilofacial, Clínica Alemana, Santiago, Chile.

${ }^{* * *}$ Jefe de Servicio. Servicio de Cirugía y Traumatología Oral y Maxilofacial, Clínica Alemana, Santiago, Chile. 
El riesgo de desarrollar ONMF aumenta con el tiempo de tratamiento y la potencia del BF. Aquellas formas de BF administradas por vía endovenosa, tienen mayor riesgo de desarrollo de ONMF que la formas orales. El BF de mayor potencia actual es el ácido zolendrónico (Gutta \& Louis). Se ha reportado que el ácido zolendrónico tiene un riesgo 9,5 veces mayor de producir ONMF que el pamidronato, fármaco que lo sigue en potencia (Novartis Pharmaceuticals Canada Inc., 2004).

Aunque la frecuencia de ONMF es incierta, la mandíbula es más frecuentemente afectada por esta patología. El maxilar está involucrado sólo entre un tercio a un cuarto de los casos (Estilo et al., 2008). Pocos estudios han sido realizados en relación a la manifestación de ONMF en el maxilar (Estilo et al.; Carlson \& Basile, 2009; Stockmann et al., 2010; Filleul et al., 2010). Con menor frecuencia aún han sido descritos casos de ONMF en el maxilar con sinusitis maxilar consecutiva (Koulocheris et al., 2008; Mast et al., 2012; Maurer et al., 2011).

La sinusitis puede ser clasificada según su etiología como rinogénica (60-80\%) u odontogénica (20$40 \%$ ). El origen odontogénico de la sinusitis puede ser a partir de un absceso periapical o de enfermedad periodontal como también procesos infecciosos consecutivos a una comunicación buco-sinusal producto de una exodoncia o el desplazamiento de objetos y/o cuerpos extraños al interior del seno, entre otros (Sandler et al., 1996). La osteonecrosis maxilar asociada al tratamiento con BF puede también provocar una sinusitis odontogénica.

La osteonecrosis maxilar avascular, no siempre está asociada a un procedimiento dental, como una exodoncia o la instalación de un implante. Ulceraciones en la mucosa oral provocadas por prótesis mal ajustadas en pacientes edéntulos o el micro daño a repetición en el hueso, producto de las fuerzas masticatorias combinadas con la inhibición del osteoclasto mediada por BF, pueden provocar osteonecrosis de los maxilares $\mathrm{y}$, consecutivamente, una sinusitis maxilar (Ruggiero et al., 2004; Koulocheris et al.; Ristow et al., 2013).

En este trabajo se presentan tres casos de pacientes con sinusitis maxilar en relación a osteonecrosis asociada a fármacos, describiendo manifestaciones clínicas, imagenológicas y el tratamiento realizado. Adicionalmente, se presenta una revisión actualizada de la literatura.

\section{REPORTE DE CASOS}

Caso 1: Mujer de 50 años de edad consulta al servicio de Cirugía Oral y Maxilofacial de Clínica Alemana de Santiago, Chile, para revisión de una herida crónica que no cicatriza en el maxilar y halitosis. La paciente desarrolló la lesión después de una exodoncia del tercer molar maxilar izquierdo, realizada por un dentista externo, 3 meses previo a la consulta en dicho centro. Como antecedente mórbido, relató cáncer de mama en estadio IV. Debido a las múltiples lesiones metastásicas óseas y hepáticas, la paciente estaba bajo tratamiento con una dosis mensual de ácido zolendrónico endovenoso por los últimos 24 meses.

Al examen clínico, se observa una área expuesta de tejido óseo necrótico en la región del tercer molar del maxilar izquierdo con supuración activa desde el alvéolo. La tomografía computadorizada de haz cónico (CBCT por sus siglas en inglés) muestra una completa obliteración del seno maxilar izquierdo (Fig. 1). El diagnóstico fue sinusitis maxilar aguda, supurativa asociada a ONMF.

Una revisión de la herida fue realizada bajo sedación endovenosa y anestesia local. Ésta fue tratada con antibióticos orales por una semana $(1 \mathrm{~g}$ de Amoxicilina cada 8 horas por 14 días) y colutorios locales con clorhexidina al $0,12 \%$ cada 8 horas por 10 días. Se indicó control después de cinco días del procedimiento o según necesidad, si alguna urgencia ocurría. La paciente no volvió para realizar un nuevo examen después del procedimiento.

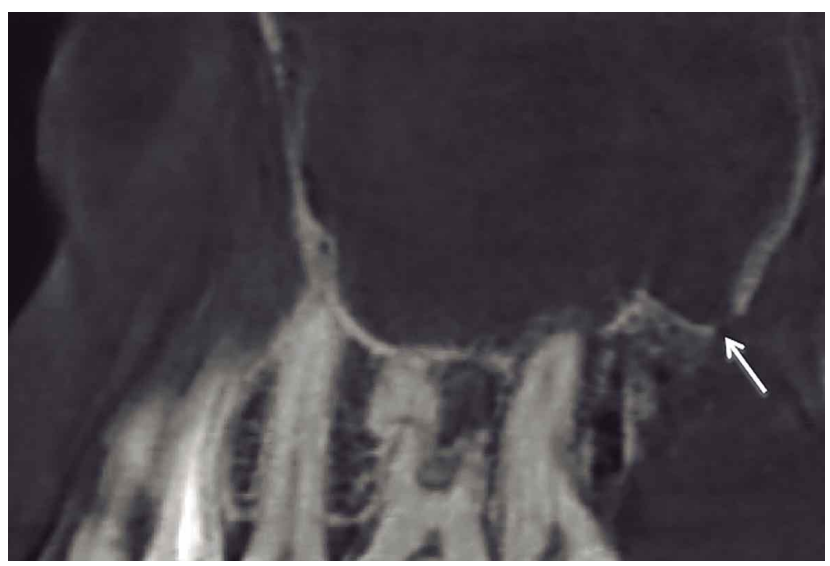

Fig. 1. Tomografia Computadorizada Cone-Beam muestra corte sagital del maxilar izquierdo. Se observa obliteración del seno maxilar y perforación de cortical (flecha). 
FIGUEROA, L.; SÁEZ, F.; ALVARADO, B.; GALLARDO, P. \& CIFUENTES, J. Sinusitis maxilar por osteonecrosis asociada a bisfosfonatos en pacientes con cáncer de mama: Serie de casos. Int. J. Odontostomat., 9(3):449-455, 2015.

Caso 2: Paciente de 41 años, sexo femenino, consulta para examen de un área expuesta de tejido óseo. Adicionalmente, consulta por aumento de volumen y dolor en zona del maxilar izquierdo y halitosis. La paciente presentaba historia de cáncer de mama. El tratamiento para el cáncer estaba basado en quimioterapia y, por los

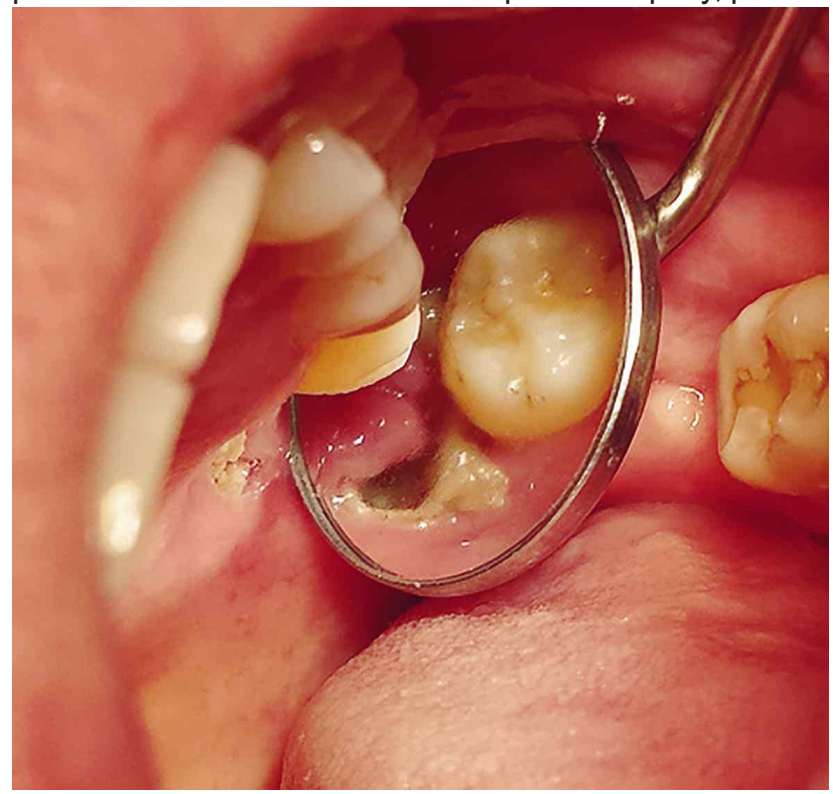

Fig. 2. Zona de tejido óseo necrótico en relación a alvéolo del tercer molar maxilar izquierdo. últimos dos años, una dosis mensual de ácido zolendrónico endovenoso. Ella desarrolló la lesión después de la extracción del tercer molar maxilar izquierdo en una consulta dental externa, dos meses antes de consultar.

Al examen clínico se observó: trismus, un área ósea expuesta en la zona palatina del alvéolo del tercer molar maxilar izquierdo, supuración por el alvéolo, eritema de la mucosa adyacente y dolor a la palpación (Fig. 2). Se solicitó un CBCT para evaluar la extensión de la necrosis. Las imágenes mostraron opacidad del seno maxilar izquierdo (Fig. 3). El diagnóstico fue: sinusitis maxilar aguda, supurativa asociada a ONMF.

La paciente fue sometida a una secuestrectomía por un cirujano maxilofacial de nuestro servicio, bajo anestesia local y sedación endovenosa. El área intervenida, fue cubierta por un colgajo muco-perióstico desplazado. Se le administró un tratamiento con Lincomicina 500 mg cada 8 horas por 7 días.

Al sexto mes de seguimiento, la lesión aún no cicatriza y la obliteración del seno es persistente según se observa en la tomografía computada realizada (Fig. 4).

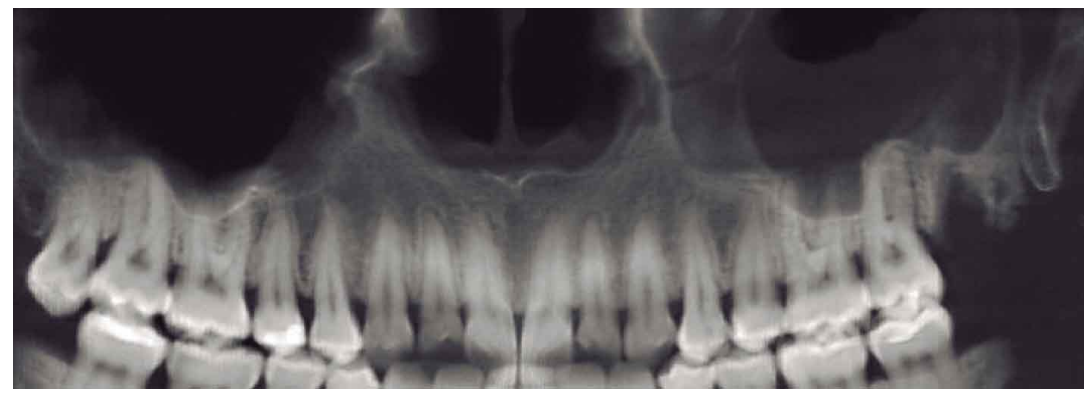

Fig. 3. Reconstrucción panorámica mediante TCCB: Completa opacificación del seno maxilar izquierdo.

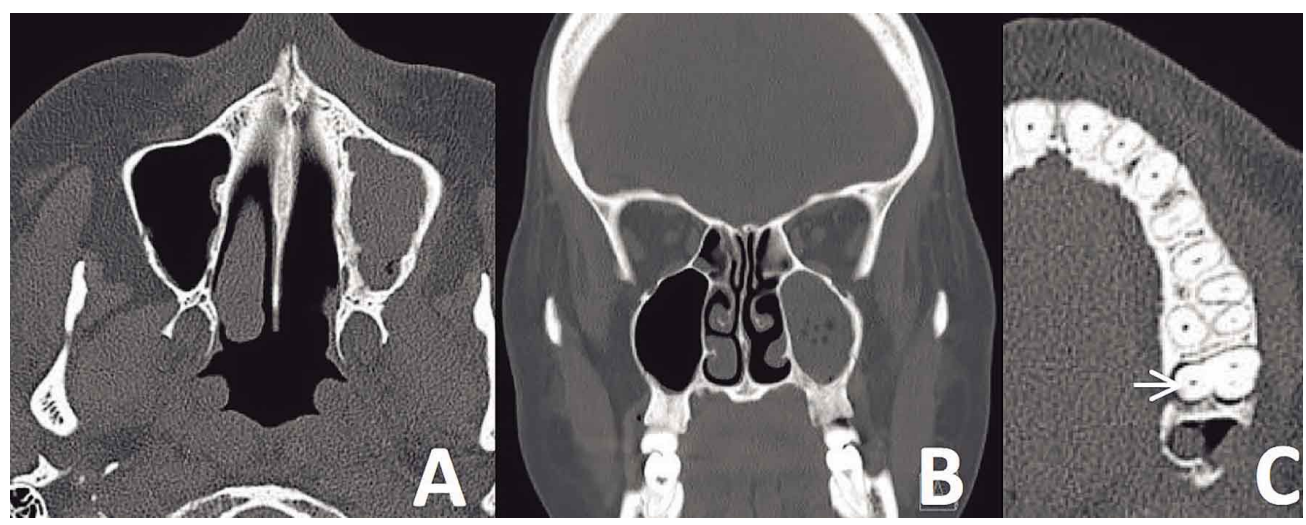

Fig. 4. Tomografia Computadorizada realizada a los 6 meses de la intervención quirúrgica. En A y B se observa una completa ocupación del seno. En C, se aprecia la comuniciación al seno maxilar a través del alvéolo dentario y el engrosamiento del ligamento periodontal en el diente adyacente (flecha). 
Caso 3: Mujer de 51 años fue derivada a nuestro servicio para evaluación de un area ósea expuesta y movilidad de dientes adyacentes en el maxilar maxilar derecho. Como antecedente mórbido, relató cáncer de mama. Estuvo en tratamiento con ibrandronato endovenoso por los últimos tres años. La lesión ósea en el maxilar se presentó nueve semanas después de realizarse de forma particular un curetaje periodontal como tratamiento de la enfermedad periodontal que presentaba. Ningún otro tratamiento dental fue descrito por la paciente.

Al examen intra-oral se observa un área de tejido óseo expuesto en relación al segundo molar maxilar derecho con pus drenando por el crévice, movilidad del primer y segundo molar que presentaban, además, tinción producto del tratamiento previo con clorhexidina. En la tomografía computada realizada, se observa un absoluto velamiento del seno maxilar derecho. Adicionalmente, se observa un ensanchamiento del ligamento periodontal del segundo molar y una comunicación con el seno maxilar a ese nivel (Fig. 5). El diagnóstico fue sinusitis maxilar asociada a ONMF.

El tratamiento propuesto fue: 1) $300 \mathrm{mg}$ de Clindamicina cada 8 horas por 7 días, 2) colutorios con Clorhexidina $0,12 \%$ tres veces al día y 3 ) Ferulización de los dientes afectados y adyacentes para disminuir la movilidad. No se planificó intervención quirúrgica debido al riesgo de aumentar el área necrótica. La paciente no acudió a control posttratamiento propuesto.

\section{DISCUSIÓN}

ONMF es más frecuente de lo que antes se asumía. Representa un efecto secundario serio que disminuye sustancialmente la calidad de vida del paciente debido al déficit funcional que produce en distintos ámbitos del sistema estomatognático como la deglución, masticación y habla, así como también el deterioro de la calidad de vida y la estética facial. Para estos pacientes es de gran importancia eliminar el dolor, controlar la infección de tejidos duros y blandos y reducir la progresión o reincidencia de la necrosis ósea (Ruggiero, 2008).

El maxilar es afectado con menor frecuencia que la mandíbula. Pocos casos de osteonecrosis asociado con sinusitis maxilar han sido publicados (Koulocheris et al.; Mast et al.; Maurer et al.). El año 2008, Koulocheris et al. reportaron dos casos de osteonecrosis inducida por BF con sinusitis maxilar supurativa como complicación. El año 2011, Maurer et al. reportaron 98 pacientes diagnosticados con ONMF, 21 de ellos con afección en el maxilar y 10 de estos a su vez, tenían sinusitis maxilar. El año 2012, Mast et al. reportaron un total de 170 pacientes con ONMF, en 53 de ellos el maxilar estaba involucrado, y sólo en uno de estos casos existía presencia de sinusitis maxilar.

La frecuencia de ONMF es mayor en pacientes con enfermedades malignas subyacentes. El mieloma múltiple y carcinoma mamario tienen mayor prevalen-

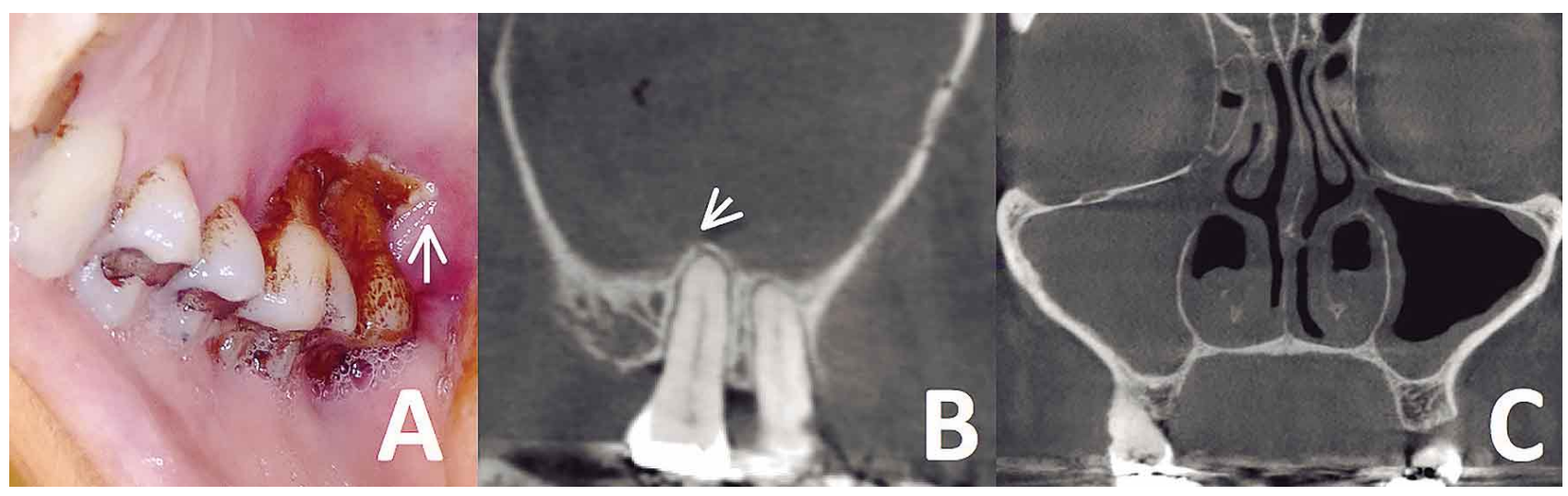

Fig.5. Caso Clínico 3. Mujer de 51 años de edad, sinusitis maxilar derecha en relación a osteonecrosis maxilar asociada a fármacos (ONMF) post curetaje periodontal. En A, pus drenando por el crevice del segundo molar (flecha) ampliamente teñido por la clorhexidina. En B, comunicación del segundo molar con el seno maxilar (punta de flecha). En B y $\mathrm{C}$ velamiento completo del seno maxilar. 
cia que la osteoporosis en el desarrollo de ONMF (Thumbigere-Math et al., 2009). En general, pacientes con enfermedades malignas, reciben BF por vía endovenosa. Estas formas de BF tienen una mayor potencia y una vida media más larga comparados con sus formas orales, resultando en una mayor acumulación del fármaco en el organismo. Por tanto, el riesgo de ONMF es mayor en pacientes con éste tipo de terapia (Maurer et al.).

El ácido zolendrónico y pamidronato están asociados a un mayor riesgo de desarrollo de ONMF que otras formas de BF8. De acuerdo con esto, en el estudio de Maurer, la mayoría de los casos reportados con ONMF $(71 \%)$ recibió una terapia con ácido zolendrónico de forma endovenosa, mientras que en el reporte de Mast et al. la misma droga fue frecuentemente asociada $(60,4 \%)$ con la aparición de esta patología.

Para este trabajo, la media de la duración del tratamiento con BF hasta la aparición de el primer signo de sinusitis fue de 28 meses; en el estudio de Mast et al., la duración de la terapia previa a la aparición de signos fue de 35,04 $\pm 13,52$ meses, y 47,4 meses en el reporte de Maurer et al.

En la regresión lineal realizada por Mast et al. se encontró que la sinusitis maxilar no fue significativamente asociada con la duración del tratamiento con BF ( $p=0,184$, odds ratio de 0,75$)$. Por otro lado, se encontró una asociación significativa entre la presencia de sinusitis activa y el desarrollo de una fistula oro-antral $(p<0,0001$, odds ratio de 20,57).

A la fecha, no existe una modalidad imagenológica idónea para el diagnóstico de ONMF. En etapas tempranas de esta patología, sólo se observa una leve osteoesclerosis y engrosamiento de la lámina dura (Hutchinson et al., 2010). Por tanto, el examen más frecuentemente solicitado es una radiografía panorámica en lugar de una imagen en 3D. Mientras que el edema de la médula ósea es mejor visualizado en una resonancia nuclear magnética, los cambios en la densidad ósea son mejor visualizados en las modalidades imagenológicas con base en los rayos X (Hohlweg-Majert et al., 2011). Si ONMF es diagnosticada en el maxilar, es de suma importancia prestar atención al estudio del compromiso del seno maxilar. Una modalidad tri-dimensional es necesaria en este caso, no solo para evaluar la extensión de la necrosis sino también para descartar el compromiso de las cavidades paranasales.
Habitualmente, la sinusitis aguda es tratada con antibióticos; sin embargo, esta terapia tiene pobres resultados una vez que ha ocurrido una osteonecrosis avascular, debido a la baja penetración que tendrán en el tejido necrótico. Por consiguiente, un procedimiento quirúrgico, como una antrotomía, puede ser necesario (Gutta \& Louis). En el estudio de Maurer et al., a ocho de los diez pacientes con sinusitis, se les realizó una antrotomía. En aquellos casos, el hueso necrótico fue removido hasta que se obtuvo sangramiento del hueso remanente. En siete casos, el defecto se cerró mediante colgajo mucoso; en un caso, se cerró con colgajo del cuerpo adiposo de la mejilla (bola de Bichat); en dos pacientes, no fue necesaria ninguna intervención quirúrgica debido a la buena respuesta a la antibioterapia endovenosa realizada. En nuestro trabajo, el primer y tercer caso no pudieron ser re-evaluados debido a que no volvieron a los controles programados. El tratamiento para la paciente del caso 2 (secuestrectomía y cierre de la comunicación con colgajo muco-perióstico más lincomicina), no fue satisfactorio. Aunque el dolor disminuyó, la deshicencia de la herida y la sinusitis maxilar persistió después de 6 meses de control.

La sinusitis purulenta es considerada como una infección de alto grado de peligrosidad en el área cráneo-facial debido a las serias complicaciones que puede traer, como: ceguera, meningitis, empiema subdural o absceso cerebral (Dazert et al., 2004). Pacientes diagnosticados con ONMF tienen, a menudo, una severa enfermedad generalizada. Por tanto, el tratamiento debería ser racional con la menor co-morbilidad posible. Pacientes con cáncer requieren, especialmente, una calidad de vida aceptable, que no debiera ser comprometida con extensos procedimientos maxilofaciales. Sin embargo, el empiema sinusal, requiere un temprano drenaje quirúrgico (Dazert et al.).

No queda claro si la sinusitis maxilar representa una patología independiente o es secundaria a la ONMF persistente en la maxila.

FIGUEROA, L.; SÁEZ, F.; ALVARADO, B.; GALLARDO, P. \& CIFUENTES, J. Maxillary sinusitis associated to bisphosphonate-related osteonecrosis in patients with breast cancer: Case series. Int. J. Odontostomat., 9(3):449-455, 2015.

ABSTRACT: Bisphosphonates are widely used as bone-stabilizers in the treatment of osseous metastases, osteoporosis, Paget's disease and others, 
due to their ability to inhibit osteoclast activity and antitumor effects. Bisphosphonate-related osteonecrosis of the jaw, nowadays medication-related osteonecrosis of the jaw (MRONJ), is defined as the presence of exposed, non-vascularized and necrotic bone tissue in the oral cavity over a period of 8 weeks with a current or previous history of treatment with antiresorptive (bisphosphonates, RANKL ligand inhibitor) and/or antiangiogenic agents, and no history of radiation therapy to the jaws or obvious metastatic disease to the jaws. The frequency of MRONJ is unclear. The mandible appears to be more frequently affected by MRONJ than the maxilla. Isolated cases of maxillary MRONJ have been described in wich a simultaneous sinusitis maxillaris was diagnosed. Three cases of MRONJ associated with maxillaris sinusitis are presented. All cases were females with a positive history of breast cancer and bisphosphonate therapy. The first two, developed MRONJ after a third molar upper extraction. The third case with MRONJ, had a history of periodontal curettage. A computed tomography was performed and showed a maxillary sinus compromise in all patients. Imaging modalities to evaluate the extent of the necrosis and the sinus compromise, as also treatment options were described in this study. Finally, an updated literature review is presented.

KEY WORDS: bisphosphonates, medicationrelated osteonecrosis, sinusitis.

\section{REFERENCIAS BIBLIOGRÁFICAS}

Abu-Id, M. H.; Warnke, P. H.; Gottschalk, J.; Springer, I.; Wiltfang, J.; Acil, Y.; Russo, P. A. \& Kreusch, T. "Bisphossy jaws" - high and low risk factors for bisphosphonate-induced osteonecrosis of the jaw. J. Craniomaxillofac. Surg., 36(2):95-103, 2008.

Carlson, E. R. \& Basile, J. D. The role of surgical resection in the management of bisphosphonate-related osteonecrosis of the jaws. J. Oral Maxillofac. Surg., 67(5 Suppl.):85-95, 2009.

Dazert, S.; Mlynski, R.; Brors, D.; Sudhoff, H. \& Prescher, A. Infection transfer between the maxillary sinus and endocranium. HNO, 52(7):631-4, 2004.

Estilo, C. L.; Van Poznak, C. H.; Williams, T.; Bohle, G. C.; Lwin, P. T.; Zhou, Q.; Riedel, E. R.; Carlson, D. L.; Schoder, H.; Farooki, A.; Fornier, M.; Halpern, J. L.; Tunick, S. J. \& Huryn, J. M. Osteonecrosis of the maxilla and mandible in patients with advanced cancer treated with bisphosphonate therapy. Oncologist, 13(8):911-20, 2008.
Filleul, O.; Crompot, E. \& Saussez, S. Bisphosphonateinduced osteonecrosis of the jaw: a review of 2,400 patient cases. J. Cancer Res. Clin. Oncol., 136(8):111724, 2010.

Gutta, R. \& Louis, P. J. Bisphosphonates and osteonecrosis of the jaws: science and rationale. Oral Surg. Oral Med. Oral Pathol. Oral Radiol. Endod., 104(2):186-93, 2007.

Hohlweg-Majert, B.; Pautke, C.; Deppe, H.; Metzger, M. C.; Wagner, K. \& Schulze, D. Qualitative and quantitative evaluation of bony structures based on DICOM dataset. J. Oral Maxillofac. Surg., 69(11):2763-70, 2011.

Hutchinson, M.; O'Ryan, F.; Chavez, V.; Lathon, P. V.; Sanchez, G.; Hatcher, D. C.; Indresano, A. T. \& Lo, J. C. Radiographic findings in bisphosphonate-treated patients with stage 0 disease in the absence of bone exposure. J. Oral Maxillofac. Surg., 68(9):2232-40, 2010.

Koulocheris, P.; Weyer, N.; Liebehenschel, N.; Otten, J. E.; Gutwald, R. \& Schmelzeisen, R. Suppurative maxillary sinusitis in patients with bisphosphonate-associated osteonecrosis of the maxilla: report of 2 cases. J. Oral Maxillofac. Surg.,66(3):539-42, 2008.

Maurer, P.; Sandulescu, T.; Kriwalsky, M. S.; Rashad, A.; Hollstein, S.; Stricker, I.; Hölzle, F. \& Kunkel, M. Bisphosphonate-related osteonecrosis of the maxilla and sinusitis maxillaris. Int. J. Oral Maxillofac. Surg., 40(3):285-91, 2011.

Mast, G.; Otto, S.; Mücke, T.; Schreyer, C.; Bissinger, O.; Kolk, A.; Wolff, K. D.; Ehrenfeld, M.; Stürzenbaum, S. R. \& Pautke, C. Incidence of maxillary sinusitis and oroantral fistulae in bisphosphonate-related osteonecrosis of the jaw. J. Craniomaxillofac. Surg., 40(7):568-71, 2012.

Novartis Pharmaceuticals Canada Inc. Update safety: possible relationship of Aredia* (pamidronate disodium) and/or Zometa* (zoledronic acid) with osteonecrosis of the jaw. Dorval, Novartis Pharmaceuticals Canada Inc., 2004. Disponible en: http://www.novartis.ca/ a $s \mathrm{k} \quad \mathrm{n}$ a $\mathrm{r} \mathrm{t}$ i $\mathrm{s} \mathrm{p} h$ a $\mathrm{r} m$ a / download.htm?res=Aredia_Zometa_DHCP E_2004_Nov.pdf\&resTitleld=249.

Reszka, A. A. \& Rodan, G. A. Mechanism of action of bisphosphonates. Curr. Osteoporos. Rep., 1(2):45-52, 2003.

Ristow, O.; Gerngroß, C.; Schwaiger, M.; Hohlweg-Majert, B.; Kehl, V.; Jansen, H.; Hahnefeld, L.; Otto, S. \& Pautke, C. Is bone turnover of jawbone and its possible over suppression by bisphosphonates of etiologic importance in pathogenesis of bisphosphonate-related osteonecrosis? J. Oral Maxillofac. Surg., 72(5):903-10, 2013. 
FIGUEROA, L.; SÁEZ, F.; ALVARADO, B.; GALLARDO, P. \& CIFUENTES, J. Sinusitis maxilar por osteonecrosis asociada a bisfosfonatos en pacientes con cáncer de mama: Serie de casos. Int. J. Odontostomat., 9(3):449-455, 2015.

Ruggiero, S. L.; Dodson, T. B.; Fantasia, J.; Goodday, R.; Aghaloo, T.; Mehrotra, B.; O'Ryan, F. \& American Association of Oral and Maxillofacial Surgeons. American Association of Oral and Maxillofacial Surgeons position paper on medication-related osteonecrosis of the jaw--2014 update. J. Oral Maxillofac. Surg., 72(10):1938-56, 2014

Ruggiero, S. L.; Dodson, T. B.; Assael, L. A.; Landesberg, R.; Marx, R. E.; Mehrotra, B. \& American Association of Oral and Maxillofacial Surgeons. American Association of Oral and Maxillofacial Surgeons position paper: Bisphosphonate-Related Osteonecrosis of the Jaws2009 update: the need to refine the BRONJ definition. J. Oral Maxillofac. Surg., 67(12):2698-9, 2009.

Ruggiero, S. L. Bisphosphonate-related osteonecrosis of the jaws. Compend. Contin. Educ. Dent., 29(2):96-8, 100-2, 104-5, 2008.

Ruggiero, S. L.; Mehrotra, B.; Rosenberg, T. J. \& Engroff, S. L. Osteonecrosis of the jaws associated with the use of bisphosphonates: a review of 63 cases. J. Oral Maxillofac. Surg., 62(5):527-34, 2004.

Sandler, N. A.; Johns, F. R. \& Braun, T. W. Advances in the management of acute and chronic sinusitis. J. Oral Maxillofac. Surg., 54(8):1005-13, 1996.

Stockmann, P.; Vairaktaris, E.; Wehrhan, F.; Seiss, M.; Schwarz, S.; Spriewald, B.; Neukam, F. W. \& Nkenke, $E$. Osteotomy and primary wound closure in bisphosphonate-associated osteonecrosis of the jaw: a prospective clinical study with 12 months follow-up. Support Care Cancer, 18(4):449-60, 2010.

Thumbigere-Math, V.; Sabino, M. C.; Gopalakrishnan, R.; Huckabay, S.; Dudek, A. Z.; Basu, S.; Hughes, P. J.; Michalowicz, B. S.; Leach, J. W.; Swenson, K. K.; Swift, J. Q.; Adkinson, C. \& Basi, D. L. Bisphosphonate-related osteonecrosis of the jaw: clinical features, risk factors, management, and treatment outcomes of 26 patients. J. Oral Maxillofac. Surg., 67(9):1904-13, 2009.
Dirección para Correspondencia:

Dr. Felipe Sáez Salas

Servicio de Cirugía y Traumatología Oral y Maxilofacial

Clínica Alemana

Santiago

CHILE

Email: fsaezsalas@gmail.com

Recibido: 13-06-2015

Aceptado: 07-10-2015 